\title{
MiR-1271 Inhibits Cell Proliferation, Invasion and EMT in Gastric Cancer by Targeting FOXQ1
}

\author{
Xiao-Jun Xiang ${ }^{a}$ Jun Deng ${ }^{a}$ Ya-Wen Liub Lu-Ying Wan ${ }^{a}$ Miao Feng ${ }^{a}$ Jun Chen ${ }^{a}$ \\ Jian-Ping Xionga
}

aDepartment of Oncology, The First Affiliated Hospital of Nanchang University, Nanchang, bDepartment of Oncology, Maternal and Child Health Hospital of Jiangxi Province, Nanchang, Jiangxi, China

\section{Key Words}

MiR-1271 • FOXQ1 • Epithelial-mesenchymal transition • Gastric cancer

\begin{abstract}
Background/Aims: FOXQ1 overexpression has been reported to enhance tumor growth and invasion. However, the biological function of FOXQ1 and the mechanism underlying its upregulation in gastric cancer (GC) remain unknown. Methods: QPCR was used to detect the expression of miR-1271 and FOXQ1 in specimens from GC patients. FOXQ1-siRNA, and miR1271 mimics and inhibitor were transfected into human MGC-803 and SGC-7901 cells. The transwell assay was used to examine the cell invasive ability. The regulation mechanism was confirmed by luciferase reporter assay. Markers of epithelial-mesenchymal transition (EMT) were detected by western blot analysis. Results: MiR-1271 was downregulated in both GC tissues and GC cell lines. The expression of miR-1271 was inversely correlated with tumor size $(P=0.017)$, tumor stage $(P=0.035)$, lymph node metastasis $(P=0.018)$, and TNM stage $(P=$ 0.025). Ectopic expression of miR-1271 dramatically suppressed GC cell proliferation, invasion, and EMT. Furthermore, FOXQ1 was identified as a direct target of miR-1271. Knockdown of FOXQ1 inhibited GC cell malignant behavior, whereas FOXQ1 overexpression partially restored the suppression effects of miR-1271. Additionally, miR-1271 expression was negatively correlated with FOXQ1 in GC tissues. Conclusions: MiR-1271 inhibits cell proliferation, invasion, and EMT in GC by directly suppressing FOXQ1 expression.
\end{abstract}

Copyright (c) 2015 S. Karger AG, Basel

\section{Introduction}

Gastric cancer (GC) is the fourth most common cancer worldwide and the second most common cause of death from cancer. Almost two thirds of the cases occur in developing

X.-J. Xiang and J. Deng contributed equally to this work.

Prof. Jian-Ping Xiong,

KARGER 125
Department of Oncology, The First Affiliated Hospital of Nanchang University, 17 Yongwaizheng Rd., Nanchang 330006 (China)

Tel. +86 79188693509 , Fax +86 79188693509 , E-Mail jpxiong_nc@sina.com 
countries and $42 \%$ in China alone [1]. Despite the rapid advancements in chemotherapy, radiation therapy, and gastric resection for GC patients, the 5-year overall survival rate has remained at about $28 \%$ owing to tumor metastasis [2,3]. To date, however, the genetic and epigenetic alterations that promote GC cell invasion and metastasis are largely unknown. Thus, the underlying molecular mechanisms remain to be elucidated.

Forkhead box protein Q1 (FOXQ1, also known as HFH1) is a member of the forkhead family of transcription factors that are expressed in different tissues and play important roles in development, metabolism, aging, and cancer [4, 5]. As one of the first forkhead genes studied, FOXQ1 has been implicated to repress the promoter activity of smooth muscle-specific genes such as telokin and SM22 $\alpha$ in A10 vascular muscle cells [6]. Recent studies have reported that overexpression of FOXQ1 is observed in different human cancers, including colorectal cancer [7], breast cancer [8], lung cancer [9, 10], and hepatocellular carcinoma $[11,12]$, and this gene upregulation enhances tumor growth and invasion $[7,8$, $10,12]$. Moreover, FOXQ1 is associated with an aggressive cancer phenotype and regulates epithelial-mesenchymal transition (EMT) by suppressing E-cadherin transcription [8, 12, 13]. In clinical settings, overexpression of FOXQ1 protein has been reported to be strongly linked to poor prognosis in cancers such as non-small cell lung cancer [9], hepatocellular carcinoma [11], and gastric cancer [14]. To date, however, the biological function of FOXQ1 in GC and the mechanism underlying its upregulation have not been examined.

MicroRNAs (miRNAs) are small, noncoding RNAs of 19-24 nucleotides in length, which suppress translation or promote the degradation of target mRNAs by directly targeting 3'-untranslated regions (3'-UTRs) [15]. Accumulating evidence indicates that abnormal miRNAs can function as oncogenes or tumor suppressors in the initiation and progression of human cancers, including GC [16-18]. For instance, miR-21 has been found to be overexpressed in GC and to promote tumor proliferation and invasion by negatively regulating important tumor suppressor genes such as PTEN, PDCD4, and RECK [19-21]. By contrast, overexpression of miR-141 could suppress GC cell invasion by directly repressing STAT4 [22]. However, it remains unclear whether miRNAs can regulate the expression of FOXQ1 in GC; therefore, we aimed to determine the target miRNAs of FOXQ1.

An miR-96 paralog, miR-1271, was found to be downregulated in GC tissues in our preliminary study. Of specific note, online bioinformatic tools indicate that miR-1271 has a conserved binding site in the FOXQ1 3'-UTR. Therefore, for the first time, we proposed and tested the hypothesis that FOXQ1 expression is downregulated by miR-1271.

\section{Materials and Methods}

Tissues and cells

A total of $60 \mathrm{GC}$ tissues and their paired normal gastric mucosal tissues were obtained in the first affiliated hospital of Nanchang University. All specimens were immediately snapped frozen in liquid nitrogen and stored at $-80^{\circ} \mathrm{C}$ until processing. This study was approved by the ethical committee of the First Affiliated Hospital of Nanchang University and carried out according to the Declaration of Helsinki, and every patient gave their written informed consent to participate in the study. The clinicopathological information is summarized in Table 1. The MGC-803, AGS, HGC-27, SGC-7901, and GES1 cell lines used were cultured in RPMI-1640 medium supplemented with 10\% fetal bovine serum (HyClone, Logan, UT, USA).

Quantitative polymerase chain reaction ( $q$ PCR) assays

Total RNA was isolated using Trizol Reagent (Invitrogen, USA), and was transcribed to cDNA using an EasyScript First-Strand cDNA Synthesis SuperMix kit (TransGen Biotech, China) according to the manufacturer's instructions. The qPCR was carried out to detect the expression of miR-1271 and FOXQ1 mRNA by using SYBR Green qPCR SuperMix-UDG with ROX (Invitrogen). The mRNA and miRNA primers were synthesized by Genepharm Company (Shanghai, China). U6 or GAPDH was used as the reference for detecting the expression of miR-1271 or FOXQ1, respectively. The primers used were as follows: miR1271-F 5'-CAG CAC TTG GCA CCT AGC A-3', miR-1271-R 5'-TAT GGT TGT TCT CCT CTC TGT CTC-3'; FOXQ1-F 
Table 1. The relationship between miR-1271 expression and clinicopathological parameters in $60 \mathrm{GC}$ patients

\begin{tabular}{|c|c|c|c|c|}
\hline \multirow[t]{2}{*}{ Characteristics } & \multirow[t]{2}{*}{ No. } & \multicolumn{2}{|c|}{ miR-1271 expression } & \multirow[t]{2}{*}{$P$ value } \\
\hline & & High expression & Low expression & \\
\hline Age (years) & & & & 0.382 \\
\hline$<60$ & 32 & 7 & 25 & \\
\hline$\geq 60$ & 28 & 8 & 20 & \\
\hline Gender & & & & 0.563 \\
\hline Male & 35 & 9 & 26 & \\
\hline Female & 25 & 6 & 19 & \\
\hline Lauren type & & & & 0.228 \\
\hline Intestinal & 33 & 10 & 23 & \\
\hline Nonintestinal & 27 & 5 & 22 & \\
\hline Differentiation & & & & 0.082 \\
\hline Low & 46 & 9 & 37 & \\
\hline Moderate or well & 14 & 6 & 8 & \\
\hline Tumor size $(\mathrm{cm})$ & & & & 0.017 \\
\hline$\leq 4$ & 24 & 10 & 14 & \\
\hline$>4$ & 36 & 5 & 31 & \\
\hline T status & & & & 0.035 \\
\hline $\mathrm{T} 1+\mathrm{T} 2$ & 12 & 6 & 6 & \\
\hline $\mathrm{T} 3+\mathrm{T} 4$ & 48 & 9 & 39 & \\
\hline N status & & & & 0.018 \\
\hline $\mathrm{N} 0+\mathrm{N} 1$ & 17 & 8 & 9 & \\
\hline $\mathrm{N} 2+\mathrm{N} 3$ & 43 & 7 & 36 & \\
\hline Distant metastasis & & & & 0.633 \\
\hline Mo & 55 & 14 & 41 & \\
\hline M1 & 5 & 1 & 4 & \\
\hline Stage & & & & 0.025 \\
\hline I-II & 25 & 10 & 15 & \\
\hline III-IV & 35 & 5 & 30 & \\
\hline
\end{tabular}

5'-GTG ATT TCT TGC TAT TGA CCG ATG-3', FOXQ1-R 5'-GCC CAA GGA GAC CAC AGT TAG A-3'; U6-F 5'-AGA GCC TGT GGT GTC CG-3', U6-R 5'-CAT CTT CAA AGC ACT TCC CT-3'; and GAPDH-F 5'-CAT CAC CAT CTT CCA GGA GCG-3', GAPDH-R 5'-TGA CCT TGC CCA CAG CCT TG-3'. Relative expression levels were calculated by the $2^{-\Delta \Delta \mathrm{Ct}}$ method.

\section{Cell transfection}

FOXQ1-siRNA (5'-UCG ACA GCA UCC UGC GCA ATT-3'), miR-1271 mimics (sense: 5'-CUU GGC ACC UAG CAA GCA CUCA-3', anti-sense: 5'-AGU GCU UGC UAG GUG CCA AGU U-3'), and an miR-1271 inhibitor (5'-UGA GUG CUU GCU AGG UGC CAA G-3') were created. The nonsense sequence vector (NC) served as a negative control. All of the vectors were synthesized by GenePharma, Shanghai. All the cells were transfected by using Lipofectamine 2000 according to the manufacturer's instructions.

\section{Cell proliferation assay}

At $24 \mathrm{~h}$ after transfection, cells were seeded into 96-well plates $\left(1 \times 10^{3}\right.$ cells/well). Cell viability was assessed by the MTT assay. The absorbance of each well was read at $490 \mathrm{~nm}$, using a spectrophotometer (Thermo Scientific). All experiments were performed three times.

Colony formation assay

After $24 \mathrm{~h}$ of transfection, cells were collected and seeded ( 300 cells/well) in a $6-\mathrm{cm}^{2}$ plate and cultured for 13 days. Effective colonies ( $>50$ cells per colony) were counted after being fixed with methanol:acetone KARGER 


\section{Cellular Physiology Cell Physiol Biochem 2015;36:1382-1394 \begin{tabular}{l|l} 
and Biochemistry Publisned onIIne: July U1, 2015 & $\begin{array}{l}\text { C) 2015 S. Karger AG, Basel } \\
\text { www.karger.com/cpb }\end{array}$ \\
\hline
\end{tabular}}

Xiang et al.: MiR-1271 Targets FOXQ1 in GC

(1:1) and stained with 5\% Gentian Violet (ICM Pharma, Singapore). All experiments were performed three times.

Transwell assay

For the transwell migration assays, MGC-803 $\left(5 \times 10^{4}\right)$ and SGC-7901 $\left(2 \times 10^{5}\right)$ cells were added to the upper chamber with a non-coated membrane (24-well insert; 8- $\mu$ m pore size; BD Biosciences) in each group, respectively. For cell invasion assays, cells were added to the upper chamber respectively, whose bottom was coated with $1 \mathrm{mg} / \mathrm{mL}$ Matrigel. Cells that invaded to the bottom surface of the insert were fixed and stained. The experiments were performed in triplicate.

Western blot analysis

Protein expression levels were analyzed by western blotting standard protocols. Briefly, $20 \mu \mathrm{L}$ of total protein extracts was resolved by denaturing sodium dodecyl sulfate-polyacrylamide gel electrophoresis and transferred to polyvinylidene difluoride membranes. The membranes were blocked with 5\% non-fat milk and then incubated with primary antibodies against FOXQ1 (ab51340; Abcam, USA), E-cadherin (AF0131; Affinity, USA), N-cadherin (ab76011; Abcam), vimentin (5741; Cell Signialing Technology, USA), GAPDH (AF7021; Affinity), and $\beta$-actin (T0022; Affinity). The blots were washed and probed with the respective secondary peroxidase-conjugated antibodies. Signals were detected using the chemiluminescence solvent (Thermo Scientific, USA).

Dual-luciferase-3'-UTR reporter assay

The potential miR-1271-binding sites were predicted by TargetScan (www.targetscan.org) and miRanda (www.microRNA.org). The luciferase-UTR reporter constructs were generated by inserting the FOXQ1 3'-UTR, carrying a putative miR-1271-binding site, into the psiCHECK2 vector (Promega), using the following primers: FOXQ1-3'UTR-HF 5'-AAT TCT AGG CGA TCG CTC GAG GAC TAC TGT TTG GGG TTT CTG G-3'; FOXQ1-3'UTR-HR 5'-GCG GCC GCT CTA GGT TTA AAC ACA CTT GCT TTC AAG GCA GTG G-3'. As a negative control, mutant FOXQ1-3'-UTR was generated by replacing GTGCCAA with CACGGTT. All PCR products were verified by DNA sequencing. GC cells were transiently co-transfected with reporter plasmids (1 $\mu \mathrm{g})$ and 50 $\mathrm{nM}$ of miR-1271 mimics or an miR-1271 inhibitor for $48 \mathrm{~h}$. The luciferase assays were performed using a luciferase assay kit (Promega) according to the manufacturer's protocol. The normalized luciferase activity was expressed as a ratio of firefly luciferase to Renilla luciferase units.

Statistical analyses

Statistical analyses were conducted using SPSS Version 18 or GraphPad Prism Version 5.0. Differences in miR-1271 and FOXQ1 mRNA expression levels between tumor tissues and matched non-tumor tissues were analyzed with the Student's t-test and analysis of variance. The correlations for miR-1271 and FOXQ1 mRNA expression were estimated using the Spearman correlation coefficient. The statistical significance of the data was determined by a Student's t-test. Differences with $P$ values of $<0.05$ were considered significant.

\section{Results}

Knockdown of FOXQ1 inhibits GC cell proliferation and invasion

To investigate the function of FOXQ1 in GC, MGC-803 cells were transfected with FOXQ1siRNA. The qPCR and western blot analyses revealed that the level of FOXQ1 in the cells was significantly decreased after the transfection (Fig. 1A). The MTT assay and colony formation assay were performed to validate the cell proliferation viability. As shown in Figure 1B and C, knockdown of FOXQ1 could suppress cell proliferation. The transwell assay showed that the silencing of FOXQ1 attenuated cell migration and invasion (Fig. 1D). Moreover, E-cadherin was upregulated and N-cadherin was downregulated in cells transfected with FOXQ1-siRNA (Fig. 1E). These results revealed that FOXQ1 is a vital oncogene in GC.

FOXQ1 is a direct target of miR-1271

To explore the upregulation mechanism of FOXQ1 in GC, we aimed to determine the target miRNAs of this gene. TargetScan and miRanda were used to find the miRNAs (Fig. 2A). 
Fig. 1. Knockdown of FOXQ1 inhibited gastric cancer cell proliferation, invasion, and epithelial-mesenchymal transition.

(A) The qPCR and western blot analyses showed that silencing of FOXQ1 decreased its mRNA and protein expression. (B) The MTT assay showed that the viability of MGC-803 cells transfected by F OXQ1 - si R N A was inhibited. (C) The colony formation assay showed that MGC-803 cells were significantly inhibited in growth after transfection with F OXQ1 - siR N A. (D) Transwell as-

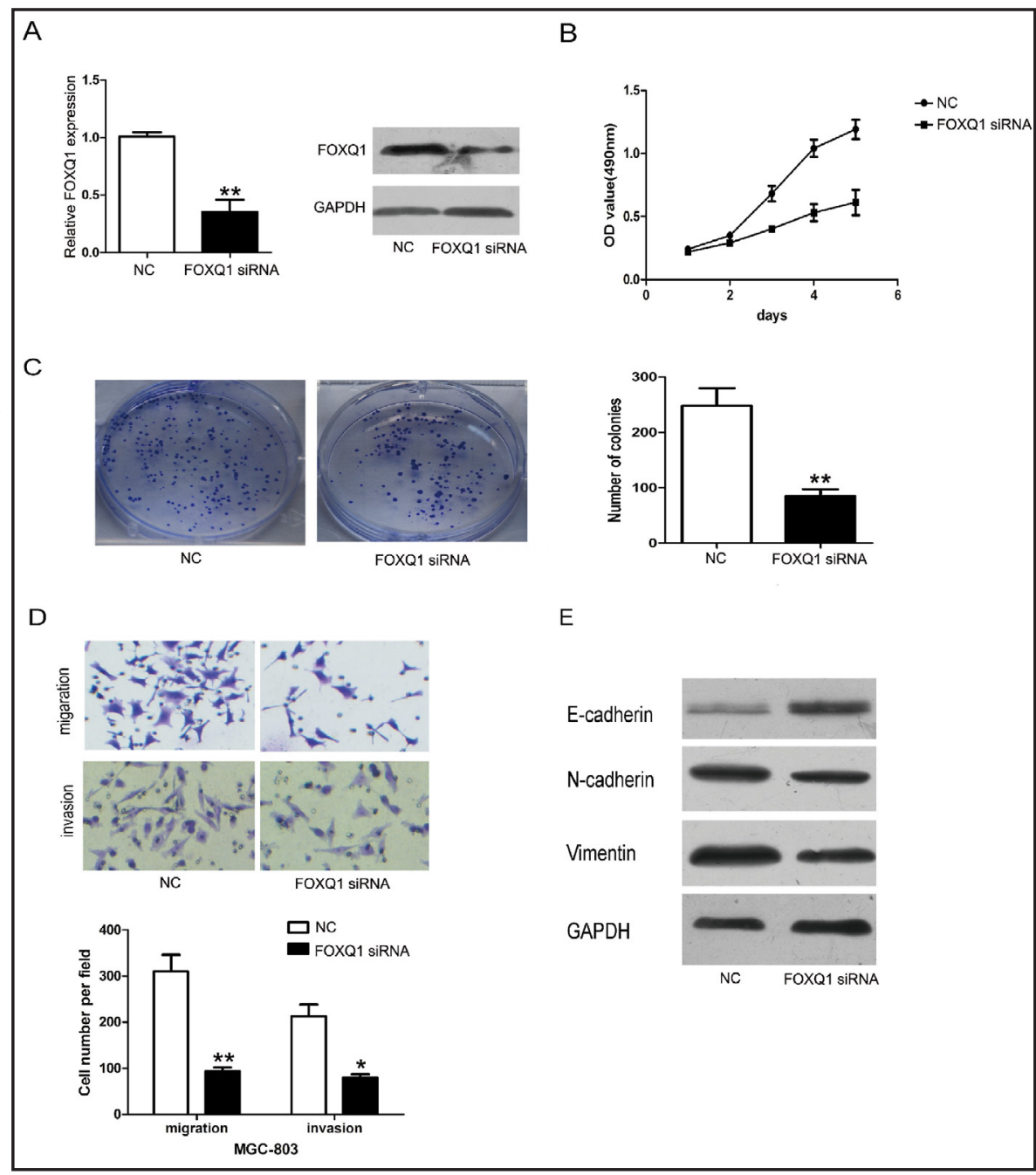

says showed that downregulation of FOXQ1 significantly inhibited cell migration and invasion compared with the negative control (NC) group. (E) Western blots showed that knockdown of FOXQ1 could upregulate E-cadherin and downregulate $\mathrm{N}$-cadherin and vimentin expression in MGC-803 cells. Three independent experiments were conducted. $* \mathrm{P}<0.05,{ }^{* *} \mathrm{P}<0.01$.

These included miR-96, miR-124, miR-9, and miR-1271. We were particularly interested in miR-1271 because of its known tumor suppressor roles in cancers. Our data showed that the co-transfection of miR-1271 significantly suppressed the firefly luciferase activity of psiCHECK2-FOXQ1-wt but failed to influence the luciferase activity of psiCHECK2-FOXQ1mut in MGC-803 cells, whereas the miR-1271 inhibitor had the opposite effect (Fig. 2B). Furthermore, upregulation of miR-1271 could decrease the mRNA and protein expression of FOXQ1, whereas miR-1271 downregulation had the opposite effect (Fig. 2C). Taken together, these results suggest that miR-1271 regulates FOXQ1 expression by directly targeting its 3'-UTR.

\section{MiR-1271 is downregulated in GC tissues}

We analyzed the expression of miR-1271 in 60 paired clinical GC tissues and their matched adjacent non-tumorous tissues by qPCR. Data are shown as $\log _{10}$ of relative ratio change of GC tissues relative to non-tumorous tissues (Fig. 3). Compared with matched nontumorous tissues, 75\% (45/60) of the GC tissues exhibited low expression of miR-1271. Moreover, the expression of miR-1271 inversely correlated with tumor size $(\mathrm{P}=0.017)$, tumor stage $(\mathrm{P}=0.035)$, lymph node metastasis $(\mathrm{P}=0.018)$, and TNM stage $(\mathrm{P}=0.025)$ 
Fig. 2. FOXQ1 is a direct target of miR-1271. (A) TargetScan (www.targetscan. org) and miRanda (www. microRNA.org) showed that FOXQ1 was a direct target of miR-1271. (B) The luciferase reporter assay showed that FOXQ1 could be targeted by miR-1271 in MGC803 and SGC-7901 cells. (C) The qPCR and western blot analyses showed that miR-1271 mimics could inhibit the mRNA and protein expression of FOXQ1 in MGC-803 cells, whereas the miR-1271 inhibitor could increase the mRNA and protein expression of FOXQ1 in SGC-7901 cells. Three independent experiments were conducted. $* \mathrm{P}<0.05,{ }^{*} \mathrm{P}<$ 0.01 .

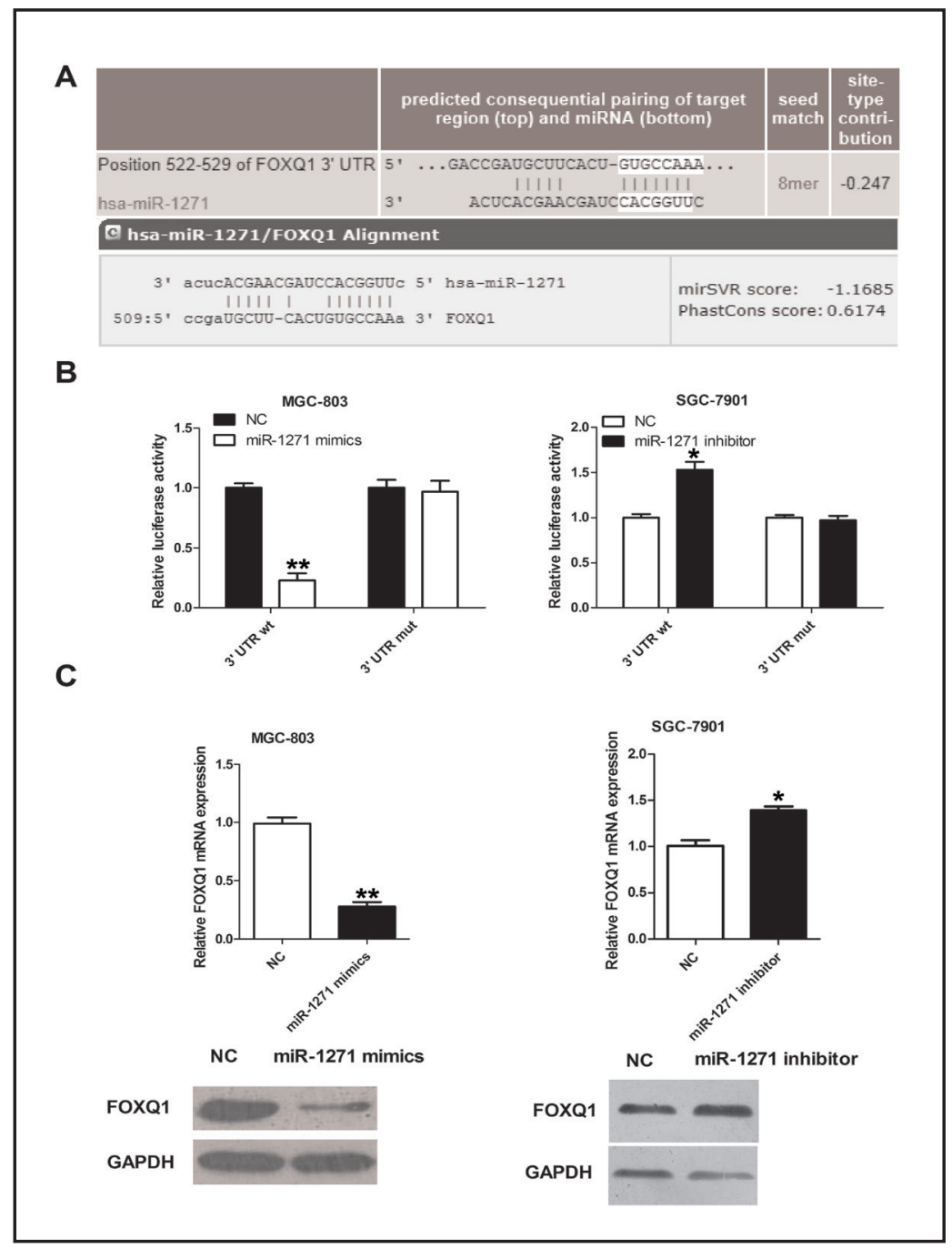

miR-1271

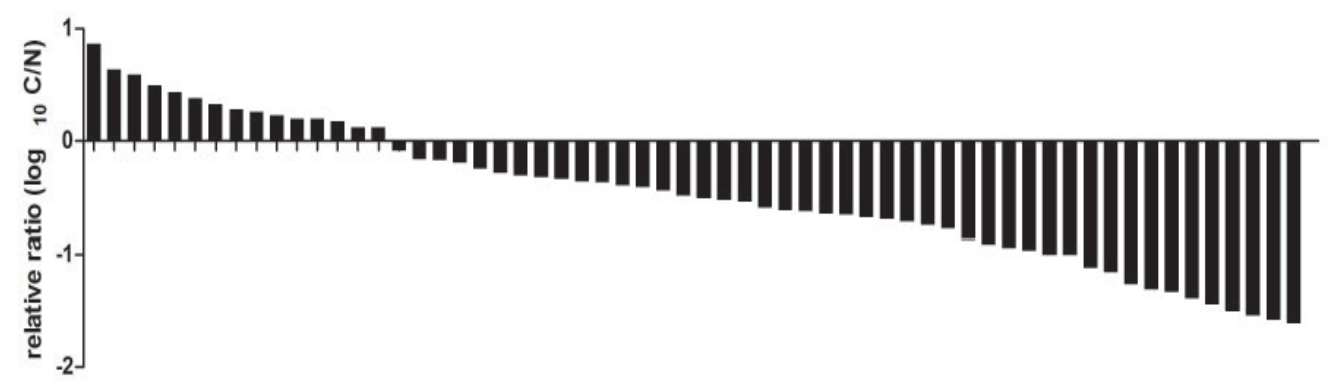

Fig. 3. MiR-1271 expression was downregulated in human gastric cancer (GC). The expression of miR-1271 in 60 pairs of GC tissues and their matched adjacent non-tumorous tissues was detected by qPCR and normalized against an endogenous control (U6 RNA). Here, data are shown as $\log _{10}$ of relative ratio change of GC tissues relative to their matched non-tumorous tissues. Compared with non-tumorous tissues, 45 cases of GC tissues exhibited low expression of miR-1271, while 15 cases showed high expression. C/N, GC tissues/ non-tumorous tissues.

(Table 1). MiR-1271 was downregulated in GC progression, indicating its potential role in suppressing the progression of GC cells.

\section{KARGER}


Fig. 4. MiR-1271 inhibited gastric cancer (GC) cell proliferation and invasion. (A) MiR-1271 was downregulated in GC cells compared with GES1 cells. (B) MiR1271 was upregulated in MGC-803 cells transfected with miR-1271 mimics. (C) The MTT assay showed that the viability of MGC-803 cells transfected with miR-1271 mimics was inhibited. (D) The colony formation assay showed that MGC-803 cells transfected with miR-1271 mimics were significantly inhibited in growth. Three independent experiments were conducted. ${ }^{*} \mathrm{P}<0.05$, ${ }^{* *} \mathrm{P}$ $<0.01$.

Fig. 5. Decreased miR1271 expression promoted SGC-7901 cell proliferation. (A) The qPCR results showed that the miR-1271 inhibitor decreased the expression level of mature miR1271 in SGC-7901 cells. (B) The MTT assay showed that the viability of SGC-7901 cells transfected with the miR-1271 inhibitor was increased. (C) The colony formation assay showed that SGC-7901 cells transfected with the miR-1271 inhibitor were significantly increased in number. Three independent experiments were conducted. ${ }^{* *} \mathrm{P}<0.01$.

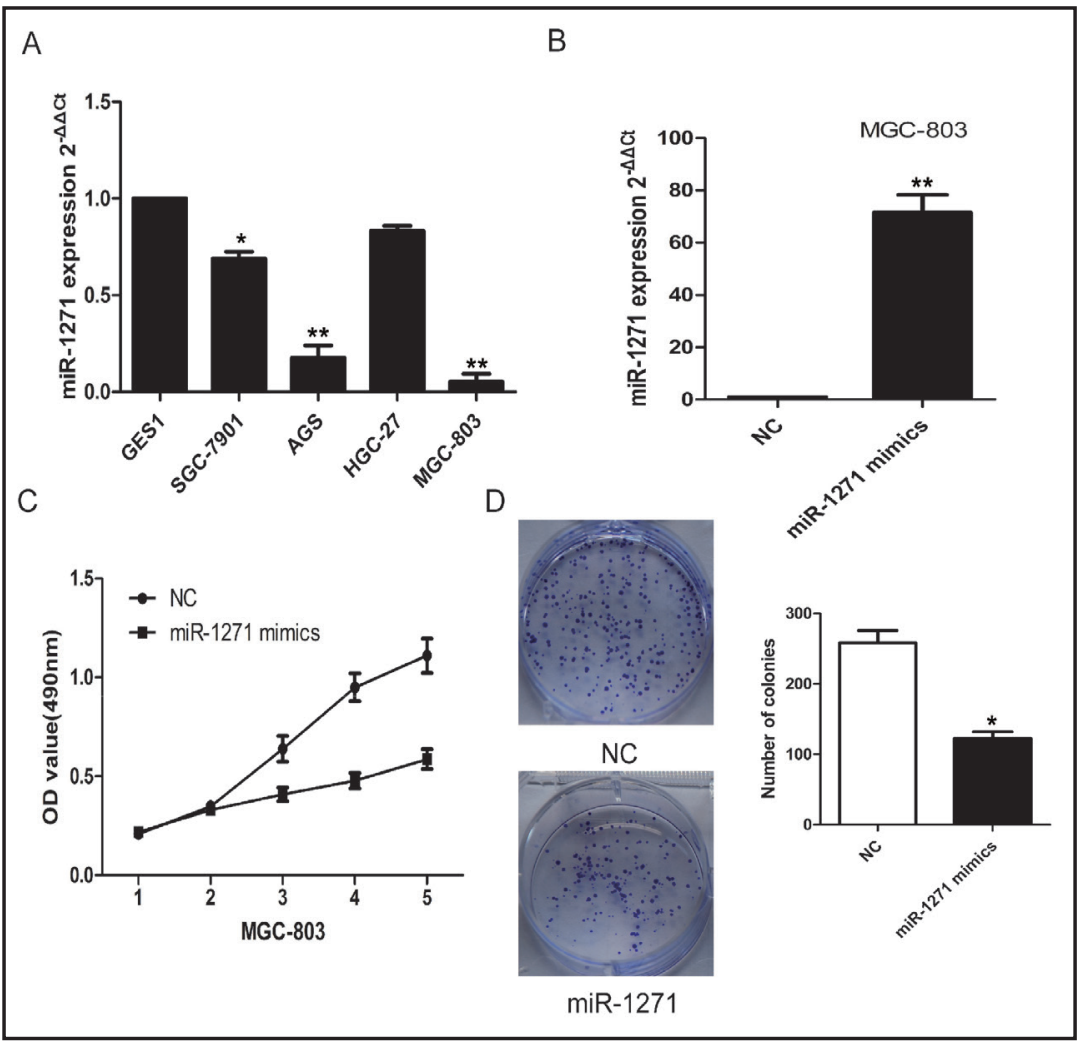

A

B
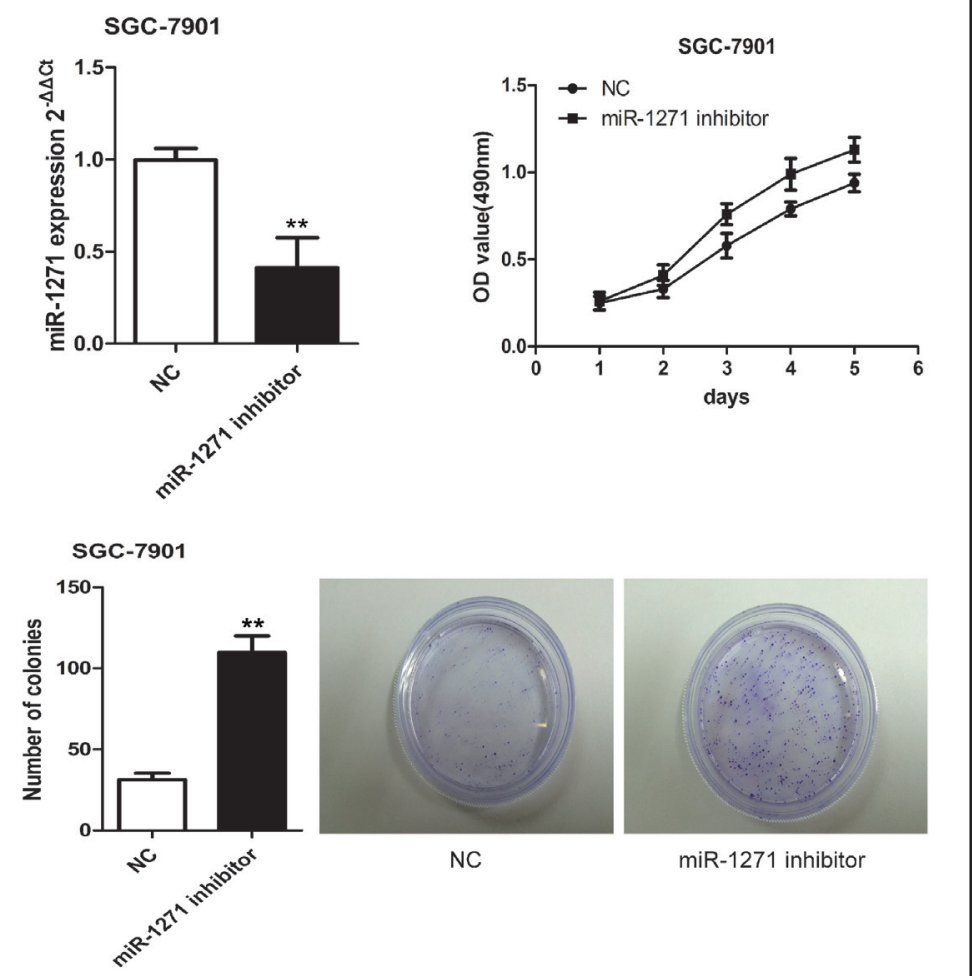

MiR-1271 inhibits GC cell proliferation and invasion

To investigate the functions of miR-1271 in GC cells, we compared its expression in GC cells with that in GES1 cells. The results showed that miR-1271 was downregulated in all 
Fig. 6. MiR-1271 inhibited gastric cancer cell migration, invasion, and epithelial-mesenchymal transition. (A) Overexpression of miR-1271 could inhibit MGC-803 cell migration and invasion, whereas its downregulation in SGC-7901 cells increased the cell migration and invasion processes. (B) Western blots showed that overexpression of miR-1271 could upregulate E-cadherin and downregulate $\mathrm{N}$-cadherin and vimentin expression in MGC-803 cells, whereas its downregulation had the opposite effect in SGC7901 cells. Three independent experiments were conducted. $* \mathrm{P}<0.05,{ }^{* *} \mathrm{P}<0.01$.

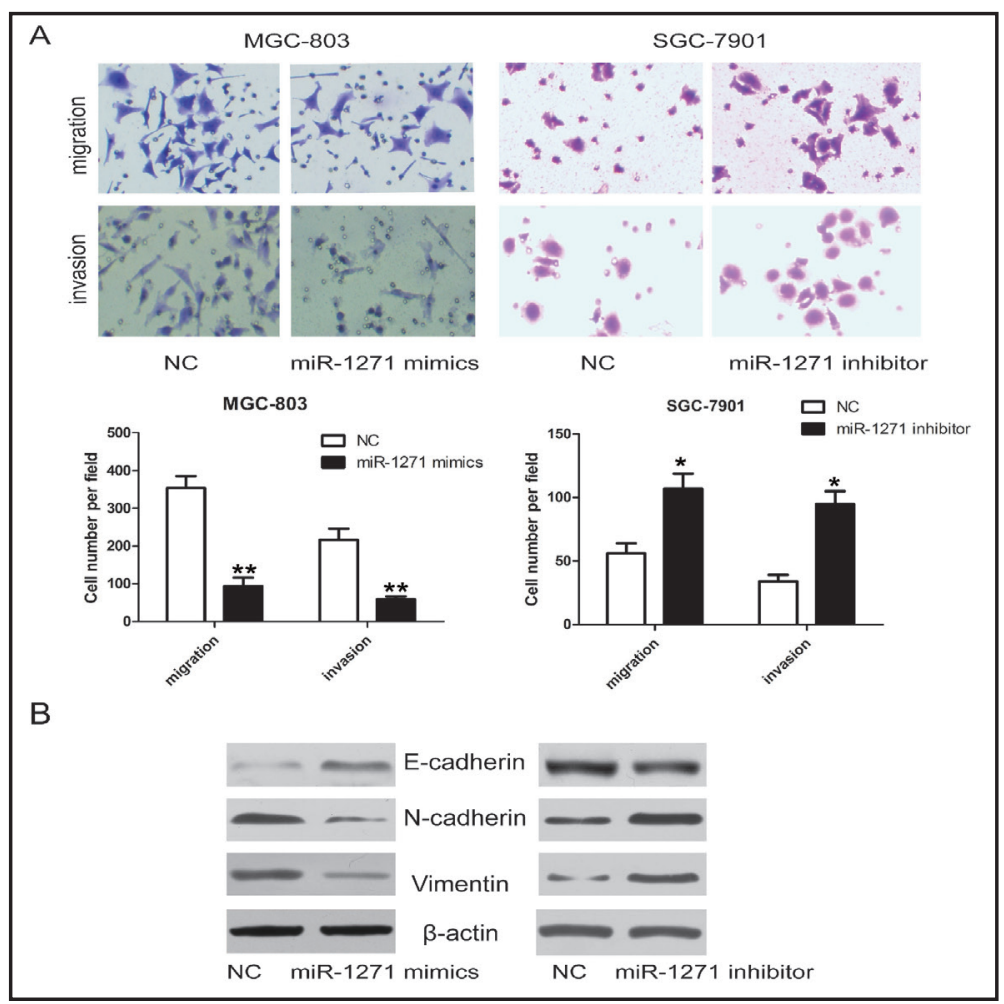

the GC cell lines (Fig. 4A). Furthermore, the expression level of miR-1271 was much lower in MGC-803 cells than that in SGC-7901 cells. Therefore, miR-1271 mimics was transfected into MGC-803 cells, and miR-1271 inhibitor was transfected into SGC-7901 cells. The qPCR results showed that miR-1271 was upregulated in MGC-803 cells transfected with the mimics (Fig. 4B), and the MTT assay and colony formation assay indicated that overexpression of miR1271 could inhibit GC cell proliferation (Fig. 4C,D). Conversly, miR-1271 was downregulated in SGC-7901 cells transfected with the inhibitor (Fig. 5A), and its downregulation could promote GC cell proliferation (Fig. 5B,C). Furthermore, the transwell assay and western blot analyses showed that overexpression of miR-1271 attenuated MGC-803 cell migration, invasion, and EMT processes (Fig. 6A,B), whereas downregulation of miR-1271 had the opposite effects in SGC-7901 cells (Fig. 6A,B). Taken together, these results suggest that miR1271 is a tumor cell suppressor in GC.

FOXQ1 overexpression partially rescues the inhibitory effects of miR-1271 on GC cells

To explore whether FOXQ1 is involved in the miR-1271-induced suppression of GC cell proliferation and invasion, we performed a rescue experiment in MGC-803 cells. A FOXQ1 overexpression construct was designed to contain only the coding sequences of FOXQ1 without its 3'-UTR (FOXQ1-no UTR). Then, miR-1271 mimics, with or without FOXQ1-no UTR construct, was transiently transfected into MGC-803 cells. As shown in Figure 7A, overexpression of FOXQ1-no UTR could partially abrogate the supressive effect mediated by miR-1271 on the protein expression of FOXQ1. The MTT assay and invasion assay showed that FOXQ1-no UTR construct could partially rescue the inhibitory effects of miR-1271 on GC cells (Fig. 7B,C). Moreover, the construct could partially rescue the inhibitory effects of miR1271 on EMT in MGC-803 cells. (Fig. 7D)

MiR-1271 is negatively correlated with FOXQ1 in GC tissues

Finally, we investigated whether FOXQ1 is upregulated in primary GC tissues and associated with miR-1271. We used qPCR to determine the FOXQ1 mRNA expression in 50 paired fresh GC and matched non-tumorous tissues. We found that FOXQ1 was upregulated in most GC tissues (Fig. 8A,C). Compared with the miR-1271 expression (Fig. 8B), statistical 
Fig. 7. Overexpression of FOXQ1 partially rescued the inhibitory effects of miR-1271 on gastric cancer (GC) cells. (A) MiR1271 mimics, or miR-1271 mimics + FOXQ1-no UTR (FOXQ1 without its 3 '-UTR) was transiently transfected into MGC-803 cells. Western blots showed that overexpression of FOXQ1no UTR could partially abrogate the supressive effect mediated by miR1271 on FOXQ1 protein expression. (B) The MTT assay showed that

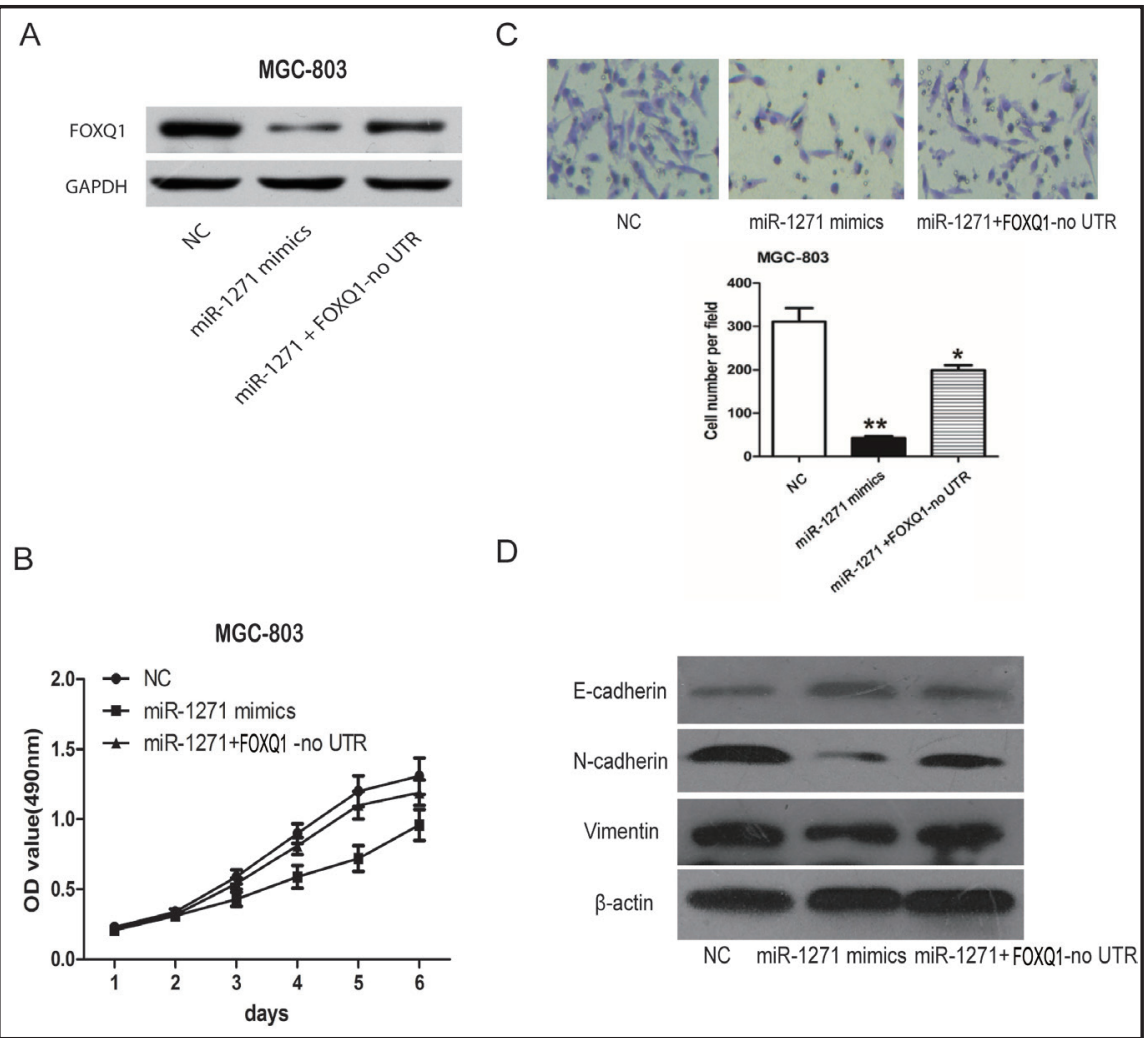
FOXQ1-no UTR partially rescued the inhibitory effects of miR-1271 on MGC-803 cells. (C) The invasion assay showed that FOXQ1-no UTR partially rescued the invasion ability of MGC-803 cells transfected with miR1271 mimics. (D) Western blots showed that overexpression of FOXQ1-no UTR partially rescued the expression of epithelial-mesenchymal transition proteins in MGC-803 cells. Three independent experiments were conducted. ${ }^{*} \mathrm{P}<0.05,{ }^{* *} \mathrm{P}<0.01$.

analysis showed that FOXQ1 levels were highly correlated with miR-1271 levels in the GC samples (Fig. 8D, $\mathrm{R}=-0.67, \mathrm{P}<0.01$ ). This result further supported that miR-1271 targets FOXQ1.

\section{Discussion}

Using bioinformatics, we predicted that the FOXQ1-targeting miRNA was miR-1271. Here, we report that miR-1271 was downregulated in both GC tissues and GC cell lines. The expression of miR-1271 was negatively associated with tumor size $(P=0.017)$, tumor stage $(\mathrm{P}=0.035)$, lymph node metastasis $(\mathrm{P}=0.018)$, and TNM stage $(\mathrm{P}=0.025)$. The ectopic expression of miR-1271 dramatically suppressed GC cell proliferation, invasion, and EMT. Furthermore, we demonstrated that miR-1271 targets FOXQ1 directly. Functional studies also showed that the knockdown of FOXQ1 inhibited GC cell proliferation, invasion, and EMT, whereas overexpression of the gene partially rescued the suppression effects of miR-1271. In addition, we found a negative correlation between miR-1271 and FOXQ1 expression in GC tissues. To the best of our knowledge, this is the first report to show that the miR-1271FOXQ1 axis regulates GC cell proliferation, invasion, and EMT in vitro.

Although FOXQ1 has been reported to be markedly overexpressed in various tumors and associated with tumor proliferation and metastasis $[7,8,10,12]$, data regarding FOXQ1 in human GC are rare. A recent study found that FOXQ1 was overexpressed in GC tissues, and this overexpression was closely related to tumor size, histological grade, lymph node 


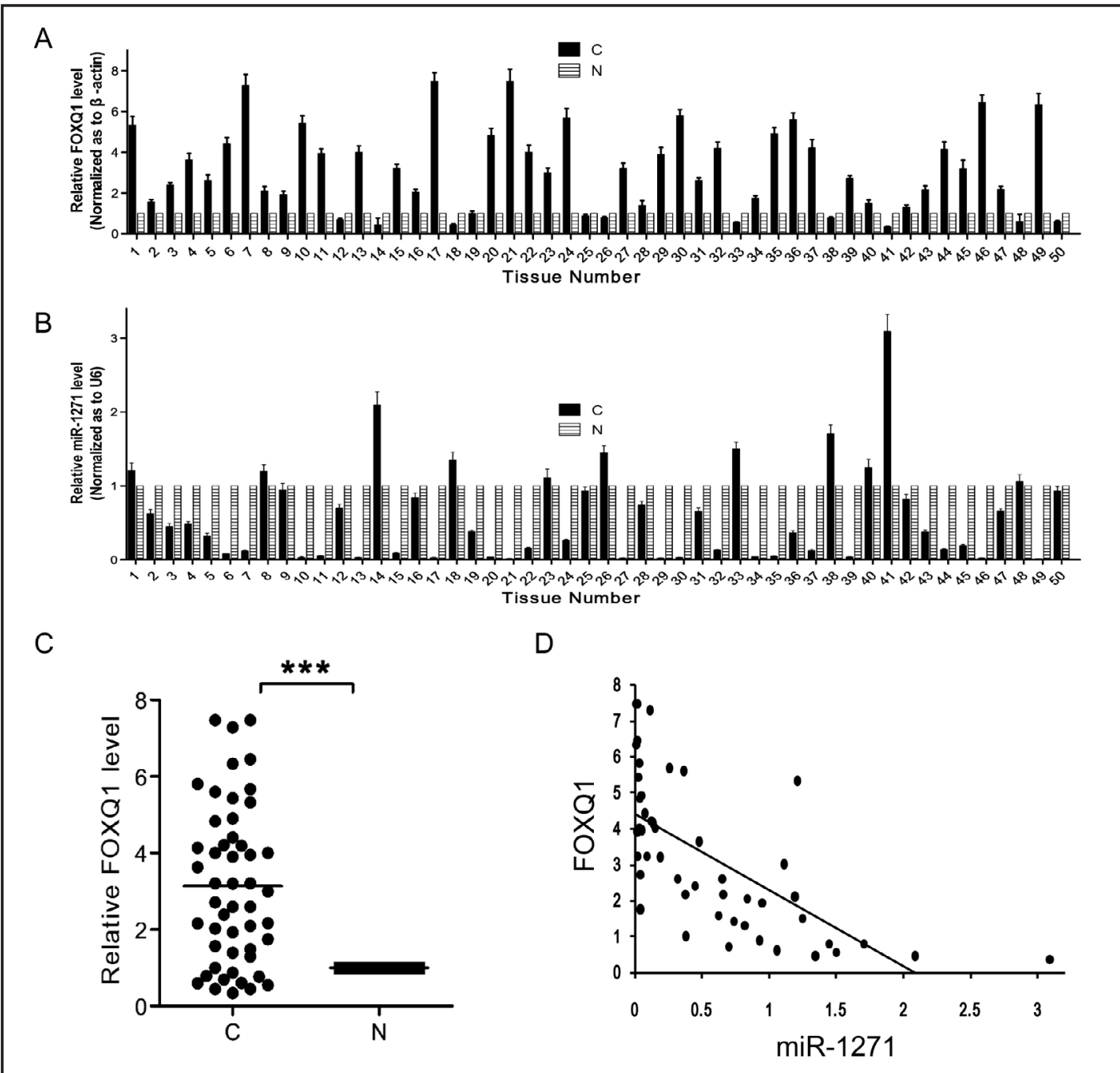

Fig. 8. MiR-1271 was negatively correlated with FOXQ1 expression in gastric cancer (GC) tissues. (A,C) The qPCR results showed that FOXQ1 was upregulated in most GC tissues compared with matched non-tumorous tissues. (B) Expression of miR-1271 in 50 matched pairs of GC tissues and their corresponding non-tumorous tissue samples. (D) Statistical analysis showed that FOXQ1 was highly correlated with the miR-1271 level in the GC samples( $\mathrm{R}=-0.67, \mathrm{P}<0.01)$. Three independent experiments were conducted. ${ }^{* * *} \mathrm{P}<0.001$.

involvement, TNM stage, and shorter overall survival of GC patients [14]. However, no further biological function of FOXQ1 in human GC has been reported. The current study demonstrates for the first time that downregulation of FOXQ1 in GC cells suppresses cell proliferation, migration/invasion, and the EMT process. Moreover, the ectopic expression of FOXQ1 in MGC-803 cells partially restored the capability of cell invasion and EMT suppressed by miR-1271. These results implied that FOXQ1 is a potential oncogene in the development and progression of GC. Nonetheless, it remains unclear how FOXQ1 contributes to the malignant behavior of GC cells. Published data on other tumors indicated the possible mechanisms as follows. First, FOXQ1 might promote cancer cell proliferation and migration by directly targeting some genes such as NRXN3 or PDGFR $\alpha$ and $\beta[23,24]$, or could exhibit an antiapoptotic effect by stimulating P21 expression [7]. Second, the FOXQ1 protein might bind directly to certain genes and induce tumor cell EMT, which is a critical event in tumor invasion and metastasis in epithelial-derived cancers. For instance, FOXQ1 expression triggered EMT by directly suppressing E-cadherin by binding to the E-box in its 
promoter region in breast cancer [8], or through the transactivation of ZEB2, TWIST1, or SOX12 expression by binding to their promoters in hepatocellular carcinoma and colorectal cancer $[12,25,26]$. Furthermore, FOXQ1 could increase angiogenesis by targeting VEGFA in colorectal cancer, and thus promote tumor growth [7]. Meanwhile, FOXQ1 also functions as a downstream target regulated by various other molecules. For example, FOXQ1 has been reported to be a target of TGF- $\beta$ signaling in breast cancer $[8,27]$, and a novel target of Wnt$\beta$-catenin pathway in colorectal cancer [28]. In addition, two recent studies indicated that FOXQ1 might be regulated by miRNAs. One study showed that miR-124 suppressed tumor growth and metastasis by targeting FOXQ1 in nasopharyngeal cancer [29]. Another study suggested that there was a double-negative feedback loop between miR-422a and its target (FOXQ1) in hepatocellular carcinoma [30]. In this study, we demonstrated for the first time that FOXQ1 was directly downregulated by miR-1271 in GC.

MiR-1271, an miR-96 paralog, encoded within the second intron of the ARL10 gene, was identified experimentally at low copy number by deep sequencing of the small RNA from human embryonic stem cells [31, 32]. Jensen et al. reported that miR-1271 was abundantly expressed in brain tissues and might play an important role in regulating aspects of neural development or function in humans [32]. To date, only a few published studies are available on its potential role in cancer development and progression. An initial study using microarray analysis indicated that miR-1271 was upregulated in patients with head and neck cancers [33]. In contrast, in a subsequent study performed by Maurel et al., miR1271 was downregulated in hepatocellular carcinoma [34]. Moreover, the downregulation of miR-1271 was associated with the concomitant upregulation of glypican-3 (GPC3), one of the most abnormally expressed genes contributing to liver carcinogenesis [34]. The findings above suggest that the expression patterns and function of miR-1271 are different in different cancers. In our study, we found that miR-1271 was commonly downregulated in both GC specimens and GC cell lines. Overexpression of miR-1271 could inhibit MGC-803 cell proliferation, migration/invasion, and the EMT process. Moreover, the opposite effects were validated in SGC-7901 cells transfected with an miR-1271 inhibitor. Our results suggest that miR-1271 is a tumor suppressor in GC, consistent with the findings in a study conducted by Yang et al. [35]. They showed that miR-1271 was downregulated in the cisplatin-resistant GC cell line SGC-7901/cisplatin. In addition, miR-1271 could regulate cisplatin resistance in human GC cells by inhibiting cell proliferation and promoting apoptosis via targeting the IGF1R/IRS1 pathway. Unlike that study, our report has demonstrated for the first time the suppressive effects of miR-1271 on GC cell migration/invasion and EMT in vitro, which are mediated, at least partially, by the direct repression of FOXQ1 expression. However, more studies are needed to further elucidate the role of miR-1271 and its associated molecular mechanism in the development and progression of GC.

In conclusion, our study identifies miR-1271 as a novel tumor suppressor that inhibits human GC cell proliferation, invasion, and EMT through repression of FOXQ1. Our findings provide evidence that miR-1271 could be a potential target for the treatment of gastric cancer.

\section{Disclosure Statement}

All authors are in agreement with content of this manuscript. The authors declare no conflicts of interest.

\section{Acknowledgements}

This study was supported by grants from the Science and Technology Department of Jiangxi Province, China (2009BSB11215, 20151BAB205039), and the Education Department of Jiangxi Province, China (GJJ 11329).

\section{KARGER}




\section{References}

1 Parkin DM, Bray F, Ferlay J, Pisani P: Global cancer statistics. CA-Cancer J Clin 2005;55:74-108.

-2 DeSantis CE, Lin CC, Mariotto AB, Siegel RL, Stein KD, Kramer JL, Alteri R, Robbins AS, Jemal A: Cancer treatment and survivorship statistics. CA-Cancer J Clin 2014;64:252-271.

- Z Zeng H, Zheng R, Guo Y, Zhang S, Zou X, Wang N, Zhang L, Tang J, Chen J, Wei K, Huang S, Wang J, Yu L, Zhao D, Song G, Chen J, Shen Y, Yang X, Gu X, Jin F, Li Q Li Y, Ge H, Zhu F, Dong J, Guo G, Wu M, Du L, Sun X, He Y, Coleman MP, Baade P, Chen W, Yu XQ: Cancer survival in china, 2003-2005: A population-based study. Int J Cancer 2014; DOI: 10.1002/ijc.29227.

-4 Feuerborn A, Srivastava PK, Kuffer S, Grandy WA, Sijmonsma TP, Gretz N, Brors B, Grone HJ: The forkhead factor FOXQ1 influences epithelial differentiation. J Cell Physiol 2011;226:710-719.

5 Jonsson H, Peng SL: Forkhead transcription factors in immunology.Cell Mol Life Sci 2005;62:397-409.

-6 Hoggatt AM, Kriegel AM, Smith AF, Herring BP: Hepatocyte nuclear factor-3 homologue 1 (hfh-1) represses transcription of smooth muscle-specific genes. J Biol Chem 2000;275:31162-31170.

7 Kaneda H, Arao T, Tanaka K, Tamura D, Aomatsu K, Kudo K, Sakai K, De Velasco MA, Matsumoto K, Fujita Y, Yamada Y, Tsurutani J, Okamoto I, Nakagawa K, Nishio K: FOXQ1 is overexpressed in colorectal cancer and enhances tumorigenicity and tumor growth. Cancer Res 2010;70:2053-2063.

-8 Zhang H, Meng F, Liu G, Zhang B, Zhu J, Wu F, Ethier SP, Miller F, Wu G: Forkhead transcription factor FOXQ1 promotes epithelial-mesenchymal transition and breast cancer metastasis. Cancer Res 2011;71:12921301.

-9 Feng J, Zhang X, Zhu H, Wang X, Ni S, Huang J: FOXQ1 overexpression influences poor prognosis in nonsmall cell lung cancer, associates with the phenomenon of emt. PloS One 2012;7:e39937.

10 Feng J, Xu L, Ni S, Gu J, Zhu H, Wang H, Zhang S, Zhang W, Huang J: Involvement of FOXQ1 in nsclc through regulating emt and increasing chemosensitivity. Oncotarget 2014;5:9689-9702.

11 Wang W, He S, Ji J, Huang J, Zhang S, Zhang Y: The prognostic significance of FOXQ1 oncogene overexpression in human hepatocellular carcinoma. Pathol Res Pract 2013;209:353-358.

12 Xia L, Huang W, Tian D, Zhang L, Qi X, Chen Z, Shang X, Nie Y, Wu K: Forkhead box q1 promotes hepatocellular carcinoma metastasis by transactivating zeb2 and versicanv1 expression. Hepatology 2014;59:958-973.

13 Qiao Y, Jiang X, Lee ST, Karuturi RK, Hooi SC, Yu Q: FOXQ1 regulates epithelial-mesenchymal transition in human cancers. Cancer Res 2011;71:3076-3086.

-14 Liang SH, Yan XZ, Wang BL, Jin HF, Yao LP, Li YN, Chen M, Nie YZ, Wang X, Guo XG, Wu KC, Ding J, Fan DM: Increased expression of FOXQ1 is a prognostic marker for patients with gastric cancer. Tumor Biol 2013;34:2605-2609.

15 Bartel DP: Micrornas: Genomics, biogenesis, mechanism, and function. Cell 2004;116:281-297.

16 Croce CM, Calin GA: Mirnas, cancer, and stem cell division. Cell 2005;122:6-7.

17 Tong F, Cao P, Yin Y, Xia S, Lai R, Liu S: Micrornas in gastric cancer: From benchtop to bedside. Digest Dis Sci 2014;59:24-30.

18 Wu WK, Lee CW, Cho CH, Fan D, Wu K, Yu J, Sung JJ: Microrna dysregulation in gastric cancer: A new player enters the game. Oncogene 2010;29:5761-5771.

-19 Yang SM, Huang C, Li XF, Yu MZ, He Y, Li J: Mir-21 confers cisplatin resistance in gastric cancer cells by regulating pten. Toxicology 2013;306:162-168.

-20 Zhang Z, Li Z, Gao C, Chen P, Chen J, Liu W, Xiao S, Lu H: Mir-21 plays a pivotal role in gastric cancer pathogenesis and progression. Lab Invest 2008;88:1358-1366.

-21 Shi Z, Zhang J, Qian X, Han L, Zhang K, Chen L, Liu J, Ren Y, Yang M, Zhang A, Pu P, Kang C: Ac1mmyr2, an inhibitor of dicer-mediated biogenesis of oncomir mir-21, reverses epithelial-mesenchymal transition and suppresses tumor growth and progression. Cancer Res 2013;73:5519-5531.

22 Zhou X, Xia Y, Su J, Zhang G: Down-regulation of miR-141 induced by helicobacter pylori promotes the invasion of gastric cancer by targeting STAT4. Cell Physiol Biochem 2014;33:1003-1012.

-23 Sun HT, Cheng SX, Tu Y, Li XH, Zhang S: FOXQ1 promotes glioma cells proliferation and migration by regulating nrxn3 expression. PloS One 2013;8:e55693.

24 Meng F, Speyer CL, Zhang B, Zhao Y, Chen W, Gorski DH, Miller FR, Wu G: PDGFR $\alpha$ and $\beta$ play critical roles in mediating Foxq1-driven breast cancer stemness and chemoresistance. Cancer Res 2015;75:584-593. 


\section{Cellular Physiology Cell Physiol Biochem 2015;36:1382-1394 \begin{tabular}{ll|l} 
and Biochemistry.1159/000430304 & $\begin{array}{l}\text { O 2015 S. Karger AG, Basel } \\
\text { www.karger.com/cpb }\end{array}$ \\
\hline Publisned onlme: July U1, 2015 &
\end{tabular} \\ Xiang et al.: MiR-1271 Targets FOXQ1 in GC}

25 Abba M, Patil N, Rasheed K, Nelson LD, Mudduluru G, Leupold JH, Allgayer H: Unraveling the role of FOXQ1 in colorectal cancer metastasis. Mol Cancer Res 2013;11:1017-1028.

-26 Huang W, Chen Z, Shang X, Tian D, Wang D, Wu K, Fan D, Xia L: Sox12, a direct target of FoxQ1, promotes hepatocellular carcinoma metastasis through up-regulating Twist1 and FGFBP1. Hepatology 2015; doi: 10.1002/hep.27756.

-27 Fan DM, Feng XS, Qi PW, Chen YW: Forkhead factor FOXQ1 promotes TGF- $\beta 1$ expression and induces epithelial-mesenchymal transition. Mol Cell Biochem 2014;397:179-186.

-28 Christensen J, Bentz S, Sengstag T, Shastri VP, Anderle P: FOXQ1, a novel target of the wnt pathway and a new marker for activation of wnt signaling in solid tumors. PloS One 2013;8:e60051.

29 Peng XH, Huang HR, Lu J, Liu X, Zhao FP, Zhang B, Lin SX, Wang L, Chen HH, Xu X, Wang F, Li XP: Mir-124 suppresses tumor growth and metastasis by targeting FOXQ1 in nasopharyngeal carcinoma. Mol Cancer 2014;13:186.

-30 Zhang J, Yang Y, Yang T, Yuan S, Wang R, Pan Z, Yang Y, Huang G, Gu F, Jiang B, Lin C, Zhou W: A doublenegative feedback loop between microrna-422a and foxg1/q1/e1 regulates hepatocellular carcinoma tumor growth and metastasis. Hepatology 2015;61:561-573.

-31 Morin RD, O'Connor MD, Griffith M, Kuchenbauer F, Delaney A, Prabhu AL, Zhao Y, McDonald H, Zeng T, Hirst M, Eaves CJ, Marra MA: Application of massively parallel sequencing to microrna profiling and discovery in human embryonic stem cells. Genome Res 2008;18:610-621.

-32 Jensen KP, Covault J: Human mir-1271 is a mir-96 paralog with distinct non-conserved brain expression pattern. Nucleic acids Res 2011;39:701-711.

-33 Nurul-Syakima AM, Yoke-Kqueen C, Sabariah AR, Shiran MS, Singh A, Learn-Han L: Differential microrna expression and identification of putative mirna targets and pathways in head and neck cancers. Int J Mol Med 2011;28:327-336.

-34 Maurel M, Jalvy S, Ladeiro Y, Combe C, Vachet L, Sagliocco F, Bioulac-Sage P, Pitard V, Jacquemin-Sablon H, Zucman-Rossi J, Laloo B, Grosset CF: A functional screening identifies five micrornas controlling glypican-3: Role of mir-1271 down-regulation in hepatocellular carcinoma. Hepatology 2013;57:195-204.

35 Yang M, Shan X, Zhou X, Qiu T, Zhu W, Ding Y, Shu Y, Liu P: Mir-1271 regulates cisplatin resistance of human gastric cancer cell lines by targeting igf1r, irs1, mtor, and bcl2. Anti-Cancer Agent Med Chem 2014;14:884-891. 